Portland State University

PDXScholar

2-1-1998

\title{
The surface plasmon enhancement effect on adsorbed molecules at elevated temperatures
}

\author{
P.T. Leung \\ Portland State University \\ Hai-Pang Chiang
}

W. S. Tse

Follow this and additional works at: https://pdxscholar.library.pdx.edu/phy_fac

Part of the Physics Commons

Let us know how access to this document benefits you.

\section{Citation Details}

Chiang, H. P., \& Leung, P. T. (1998). The surface plasmon enhancement effect on adsorbed molecules at elevated temperatures. Journal Of Chemical Physics, 108(6), 2659.

This Article is brought to you for free and open access. It has been accepted for inclusion in Physics Faculty Publications and Presentations by an authorized administrator of PDXScholar. Please contact us if we can make this document more accessible: pdxscholar@pdx.edu. 


\title{
LETTERS TO THE EDITOR
}

The Letters to the Editor section is divided into three categories entitled Notes, Comments, and Errata. Letters to the Editor are limited to one and three-fourths journal pages as described in the Announcement in the 1 January 1998 issue.

\section{NOTES}

\section{The surface plasmon enhancement effect on adsorbed molecules at elevated temperatures}

\author{
H.-P. Chiang, P. T. Leung, ${ }^{\text {a) }}$ and W. S. Tse \\ Institute of Physics, Academia Sinica, Taipei 11529, Taiwan, Republic of China
}

(Received 28 August 1997; accepted 31 October 1997)

[S0021-9606(98)01106-4]

Ever since the first observation of surface enhanced Raman scattering (SERS) in 1974, ${ }^{1}$ the possibility of enhancing other processes, such as photodissocation at rough metallic surfaces, has been seriously studied. ${ }^{2}$ Although the complete detailed mechanism for SERS has yet to be understood, it is widely accepted that a major contribution arises from the excitation of the surface plasmon resonance (SPR) field which can couple to the radiation field in the presence of surface roughness. ${ }^{3}$ Since this mechanism is independent of the details of the molecular process, it is thus expected that other processes in photochemistry can also be enhanced at a rough metal surface. Moreover, it often happens that realistic catalytic conditions for these processes require a high substrate temperature. It is thus of interest to investigate the possibility of sustaining such a SPR-enhancement mechanism at elevated temperatures. Though one has to acknowledge that many other mechanisms (e.g., surface phonon effects) and factors (e.g., surface morphology) could be of relevance to the surface enhancements observed, we single out the SPR as a prominent effect recognized (at least at room temperature) in the hope that our modeling results will stimulate experimentalist for a systematic study of this problem. We shall use SERS as an example in our present study.

Recent preliminary observation of SERS from chemisorbed oxygen ${ }^{4}$ at silver surfaces to take place up to $900^{\circ} \mathrm{K}$ has further stimulated studies in this direction. Previously, we adopted a simplified model for a first attempt to understand the phenomenon theoretically. ${ }^{5}$ In particular, we have modeled the surface roughness as a spherical island with a local dielectric response, and the substrate optical properties are accounted for using a temperature-dependent collision frequency in the Drude model as first treated by Ujihara. ${ }^{6}$ However, the Debye model for the electron-phonon collision frequency was not handled in a consistent manner. ${ }^{5,6}$ Furthermore, it has been shown that nonlocal electrodynamic effects arising from the "quantum spread" of the surface electrons are very significant at close molecule-surface distances. ${ }^{7,8}$ Hence, it is the purpose here to present a much improved model for a theoretical study of the problem. The present model includes a more consistent treatment of the temperature variation of the phonon-electron collision, as well as the electron-electron collision contribution to the collision frequency in the Drude model. ${ }^{9}$ Although it is known that the Drude model has only limited applicability for noble metals within a certain range of frequencies in which electronic intraband transitions dominate, we expect that the SPR effect should be well accounted for since it involves mainly collective motions of the free electrons. We shall also take into account the nonlocal dielectric response of the substrate metal.

As a model study, we consider the Gersten-Nitzan (GN) model $^{10}$ of a dipole-sphere system for SERS from which one obtains the enhancement ratio as:

$$
R=\left|\frac{1}{1-\alpha G_{\perp}}\left[1+\frac{2 \alpha_{1}^{S}}{(a+d)^{3}}\right]\right|^{4},
$$

where $\alpha$ is the molecular polarizability (taken as $10 \AA^{3}$ ), $a$ the radius, and $d$ the molecule-sphere distance, respectively, and $G_{\perp}$ is the "image-field factor" which is a function of the $n$th pole polarizability $\left(\alpha_{n}^{S}\right)$ of the sphere. ${ }^{11}$ Thus the "surface roughness" is modeled in a highly simplified way in terms of isolated and nondisperse particles, which can simulate experimental situation using a "specially prepared" island surface. ${ }^{12}$ The original GN model assumed a local dielectric response function $\epsilon(\omega)$ entering $\alpha_{n}^{S}$. Previously, we studied the nonlocal effects on $\alpha_{n}^{S}$ using a wave vector dependent function $\epsilon(\mathbf{k}, \omega)$ which can be expressed in terms of $\epsilon(\omega)$ within the hydrodynamic model. ${ }^{13}$ We have found significant nonlocal effects for SERS at $d \leqslant 10 \mathrm{~nm}^{8,14}$ Hence the following study will include these effects.

The dominant temperature dependence of $R$ in Eq. (1) will enter through $\alpha_{n}^{S}$ with the introduction of a temperaturedependent Drude function as follows:

$$
\epsilon(T)=1-\frac{\omega_{p}^{2}}{\omega\left(\omega+i \omega_{c}(T)\right)},
$$

where we have assumed the minor temperature dependence of the plasmon frequency $\omega_{p}$ to be negligible. ${ }^{5}$ The tempera- 


\section{R vs T}

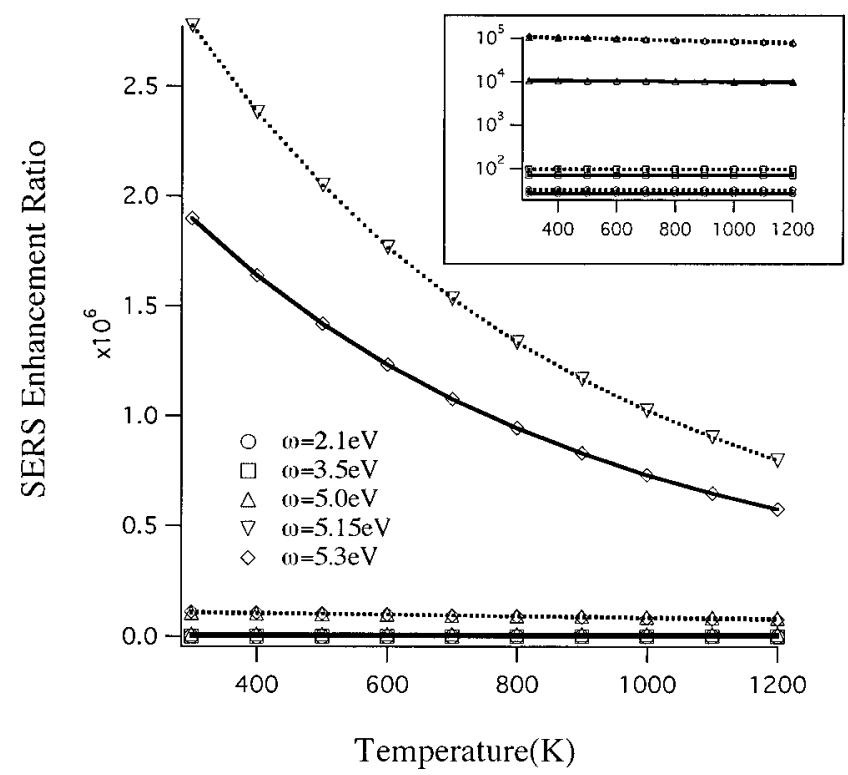

FIG. 1. SERS enhancement ratio as a function of substrate temperature at different scattering frequencies according to both the nonlocal (solid line) and local (dotted line) models.

ture dependence of the collision frequency $\omega_{c}$, including the contributions from both phonon-electron and electronelectron scattering, can be obtained as: ${ }^{9}$

$$
\omega_{c}=\omega_{c p}+\omega_{c e},
$$

with $\omega_{c p}$ given by the Holstein model,

$$
\omega_{c p}(T)=\omega_{0}\left[\frac{2}{5}+4\left(\frac{T}{\theta}\right)^{5} \int_{0}^{\theta / T} \frac{z^{4}}{e^{z}-1} d z\right]
$$

where $\theta$ is the Debye temperature and the constant $\omega_{0}$ must be determined with care. ${ }^{9}$ This last step was not treated in a consistent way previously. ${ }^{5,6}$ Furthermore, the "zero-point frequency" in (4) is found to remain significant up to the melting temperature of the metal $(\approx 4 \%$ contribution for silver at $T=1200 \mathrm{~K}$ ), but was ignored in the previous models. ${ }^{5,6} \omega_{c e}$ in Eq. (3) used here is given by the Lawrence result ${ }^{15}$ in the following form:

$$
\omega_{c e}=\frac{1}{12} \pi^{3} \frac{\Gamma \Delta}{\hbar E_{F}}\left[\left(k_{B} T\right)^{2}+\left(\frac{\hbar \omega}{2 \pi}\right)^{2}\right],
$$

where $E_{F}$ is the Fermi energy, $k_{B}$ the Boltzmann constant, $\Gamma$ a constant averaging the scattering probability over the Fermi surface, and $\Delta$ the fractional umklapp scattering.

We have performed a numerical study of the temperature effects on SERS using the above improved model taking silver as the substrate. The parameters required can be found from Ref. 9. The molecule-surface distance and the sphere radius are fixed at 10 and $50 \AA$, respectively. Figure 1 shows the enhancement ratio as a function of temperature for different scattering frequencies. It is seen that the decrease in $R$ as $T$ increases is only significant for frequencies close to that of the surface plasmon resonance of the substrate (at $\omega \approx 5$ $\mathrm{eV}$ ). The insignificant variation of $R$ with $T$ for "offresonant" scattering frequencies can be seen more clearly from a logarithmic plot shown in the inset. While these results are qualitatively comparable with those observed previously using a cruder model, ${ }^{5}$ the decrease in $R$ is here quantitatively much milder as temperature increases, even for scattering close to the SPR frequency of the substrate. In particular, no "crossing" between the $R$ vs $T$ curve is observed as was seen before. ${ }^{5}$ Furthermore, one sees that the nonlocal effect will in general lead to both a lower value and a lower rate of decrease for $R$ as a function of temperature.

Hence, according to the present modeling results, it appears that the SPR enhancement mechanism can most of the time survive at high substrate temperatures. Of course, consideration of more realistic surface morphology and other physical mechanisms can change our results dramatically. However, if this result can be confirmed experimentally, then not only SERS but also other spectroscopic and catalytic photochemical processes can be enhanced at elevated substrate temperatures. We thus believe a definitive experimental clarification of this issue over a large range of frequencies will be desirable, given the complexity and various idealizations of the theoretical modeling of the problem.

P.T.L. acknowledges the support of the Institute of Physics provided during his stay.

a) Author to whom correspondence should be addressed; permanent address: Department of Physics, Portland State University, P. O. Box 751, Portland, OR 97207-0751.

${ }^{1}$ M. Fleischmann, P. J. Handra, and A. J. MaQuillan, Chem. Phys. Lett. 26, 163 (1974).

${ }^{2}$ D. A. Jelski, P. T. Leung, and T. F. George, Int. Rev. Phys. Chem. 7, 179 (1988).

${ }^{3}$ See, e.g., M. Moskovits, Rev. Mod. Phys. 57, 783 (1985).

${ }^{4}$ B. Pettinger, X. Boa, I. C. Wilcock, M. Muhler, and G. Ertl, Phys. Rev. Lett. 72, 1561 (1994).

${ }^{5}$ P. T. Leung, M. H. Hider, and E. J. Sanchez, Phys. Rev. B 53, 12659 (1996).

${ }^{6}$ K. Ujihara, J. Appl. Phys. 43, 2376 (1972).

${ }^{7}$ W. H. Weber and G. W. Ford, Phys. Rev. Lett. 44, 1774 (1980).

${ }^{8}$ P. T. Leung and W. S. Tse, Solid State Commun. 95, 39 (1995).

${ }^{9}$ H.-P. Chiang, P. T. Leung, and W. S. Tse, Solid State Commun. 101, 45 (1997).

${ }^{10}$ J. Gersten and A. Nitzan, J. Chem. Phys. 73, 3023 (1980).

${ }^{11}$ See Refs. 5 and 10 for more complicated nonspherical surface structures.

${ }^{12}$ R. Koh, S. Haayashi, and K. Yamamoto, Solid State Commun. 64, 375 (1987). See also Ref. 8 for a scanning tunneling microscopy (STM) technique discussed there, which enables one to prepare well-defined surface morphology with isolated almost-spherical islands.

${ }^{13}$ R. Fuchs and F. Claro, Phys. Rev. B 35, 3722 (1987).

${ }^{14}$ See also, O. Stenzel, A. Stendal, D. Drews, T. Werninghaus, M. Falke, D. R. T. Zahn, C. von Borczyskowski, Appl. Surf. Sci. 108, 71 (1997).

${ }^{15}$ W. E. Lawrence, Phys. Rev. B 13, 5316 (1976). 